\title{
Area dependence and influence of crystal inhomogeneity on superconducting properties of Bi2212 mesa structures
}

\author{
Y. Demirhan a, *, H. Saglam a, F. Turkoglu a, H. Alaboz a, L. Ozyuzer a , N. Miyakawa ${ }^{\text {b }}$, \\ K. Kadowaki ${ }^{\mathrm{c}}$ \\ a Department of Physics, Izmir Institute of Technology, Urla 35430, Izmir, Turkey \\ ${ }^{\mathrm{b}}$ Department of Applied Physics, Tokyo University of Science, Tokyo, Japan \\ c University of Tsukuba, Tsukuba, Japan
}

\section{A R T I C L E I N F O}

\section{Article history:}

Received 1 December 2014

Received in revised form 2 May 2015

Accepted 7 May 2015

Available online 16 May 2015

\section{Keywords:}

Terahertz emitters

Intrinsic Josephson junctions

Bi2212 single crystals

ac-Josephson effect

High- $\mathrm{T}_{\mathrm{c}}$ superconductors

\begin{abstract}
A B S T R A C
The rapid increase in applications of terahertz waves requires new techniques to obtain continuous wave terahertz sources. Mesa structures fabricated from high- $\mathrm{T}_{\mathrm{c}}$ superconductor $\mathrm{Bi}_{2} \mathrm{Sr}_{2} \mathrm{CaCu}_{2} \mathrm{O}_{8+\delta}(\mathrm{Bi} 2212)$ single crystal have been observed as an intense, coherent, continuous electromagnetic wave source in the terahertz $(\mathrm{THz})$ frequency region. However, in order to produce coherent radiation with high applicable power, we need large mesa structures that enter a collective electromagnetic state in which their oscillations are largely synchronized in phase. On the other hand, large mesa structures cause a heating problem. In this study, we report on the critical current density dependence of mesa area and the crystal inhomogeneity to understand heating problems in large area mesas for terahertz radiation. Since the doping dependence of $\mathrm{Bi} 2212$ is an important parameter, the as-grown $\mathrm{Bi} 2212$ crystals were heat-treated at various temperatures under vacuum conditions. We have fabricated triple mesa structures from Bi2212 single crystal using e-beam lithography and argon ion beam etching techniques with same area and with different area on the same chip. We investigated and compared characteristics of triple mesas which are on the same chip and next to each other. In this way, we searched the crystal inhomogeneity in triple mesa structures and studied the critical current density dependence of mesa area to obtain high emission power for the $\mathrm{THz}$ radiation. Our experimental results clearly show that the Josephson critical current density is decreasing when the area of mesa is increasing.
\end{abstract}

(c) 2015 Elsevier Ltd. All rights reserved.

\section{Introduction}

Applications of electromagnetic waves in the under-developed terahertz frequency range are rapidly increasing and a wellunderstood technique of efficient terahertz $(\mathrm{THz})$ wave generation is needed [1-4]. As an extremely attractive field of research, terahertz technology still needs compact solid-state sources. All application areas, such as imaging, spectroscopy, information technology, medical diagnosis [5] will take advantage from the improvement of $\mathrm{THz}$ source efficiency. The investigation of coherent $\mathrm{THz}$ emission from stacks of intrinsic Josephson junctions (IJJs) produced from the high temperature superconductor $\mathrm{Bi}_{2} \mathrm{Sr}_{2}$ $\mathrm{CaCu}_{2} \mathrm{O}_{8}(\mathrm{Bi2212})$ has come forward as a primary research field in

\footnotetext{
* Corresponding author. Tel.: +90 2327507683.

E-mail address: yasemindemirhan@iyte.edu.tr (Y. Demirhan).
}

recent years, both from experimental and theoretical points of view [6-13].

When a dc voltage is applied across a Josephson junction, a superconducting a.c. current will flow at the Josephson frequency, which is given by the a.c. Josephson relation, $f=\left(2 \pi / \Phi_{0}\right) \times V_{\text {jun }}$, where $\Phi_{0}=2.07 \times 10^{-15} \mathrm{~V} \mathrm{~s}$ is the magnetic flux quantum and $V_{j u n}$ is the junction voltage [14]. Therefore, with a voltage of $1 \mathrm{mV}$ corresponding to a frequency of $0.4836 \mathrm{THz}$, a Josephson junction is a natural converter of a dc voltage into a high-frequency current. The frequency and the junction voltage proportionality is a promising fact for the design of frequency-tunable radiation sources. Ozyuzer et al. successfully [6] observed directly the strong emission of electromagnetic waves using a mesa of Bi2212 crystal in the absence of an external magnetic field; the mesa shaped $\mathrm{THz}$ device with sizable power works as a dc voltage to high frequency converter.

In $\mathrm{THz}$ emission studies of superconducting mesas, various shapes of $\mathrm{Bi} 2212$ resonators are introduced. Resonators with almost 
rectangular shape are patterned onto Bi2212 crystals by e-beam lithography and ion beam etching techniques [15-18]. The resonators have typical dimensions of $\sim 1-2 \mu \mathrm{m}$ in height (corresponding to roughly $1000 \mathrm{IJJs}$ ), $\sim 40-100 \mu \mathrm{m}$ in width and several hundreds of micrometers in length. In our previous papers [19-21]; it is feasible to synchronize more than $\mathrm{N}=650$ junctions to oscillate in phase, thus generating continuous and coherent radiation with power up to $60 \mu \mathrm{W}$ at emission frequencies ranging from 0.36 to $0.85 \mathrm{THz}$ which is inversely proportional to the mesa width from 100 to $40 \mu \mathrm{m}$. We think that an electromagnetic cavity is generated by the mesa and in this cavity an alternating electromagnetic field is stored. Then in the individual junctions the cavity resonance synchronizes the Josephson oscillations.

A stack of coupled IJJs have a very complex dynamical behavior and therefore the exact mechanism is questionable. A very important stage in realization of the mechanism is hot spot detection by low temperature laser scanning microscopy (LSTEM) which plays an important role. The existence of wave structures in the stacks is verified by LSTEM [22-24] and, apart from this, the formation of electrothermal domains in such structures is observed. In the experiments on LSTEM where a modulated laser pulse induces a local variation in the c-axis resistivity and/or critical current of Bi2212 is used, it is shown that the self-heating is not uniform and takes the form of one or more localized hot spots. Therefore, to understand the interaction between non-uniform temperature and electromagnetic modes is very important in order to design of Bi2212 mesas capable of generating technologically interesting levels of $\mathrm{THz}$ power. With the purpose of demonstrating the mechanism of the $\mathrm{THz}$ emission from an $\mathrm{IJJ}$, a comprehensive investigation of temperature and bias dependence of the emission spectrum is insignificant. The thermal conductivity along the c-axis is poor and, consequently, large $\mathrm{Bi} 2212$ mesas for $\mathrm{THz}$ emission are vulnerable to self-heating. The $\mathrm{THz}$ emission properties are considerably affected by this heating phenomenon, so it is very important to get control over its behavior [25,26].

The Joule-heating-induced temperature rise in an IJJ stack can be described by a ballistic phonon transport model proposed by Krasnov et al. [26,27];

$\Delta \mathrm{T}_{\text {ball }} \propto \mathrm{aP} / \kappa_{\mathrm{ab}} \mathrm{I}_{\mathrm{ph}}$

where $\mathrm{P}=\mathrm{JV}$ is the generated power per area inside the stack, $a$ is a constant to be determined, $\kappa_{\mathrm{ab}}$ is in-plane thermal conductivity and $\mathrm{I}_{\mathrm{ph}}$ is phonon mean free path.

A new operation mode for $\mathrm{Bi} 2212 \mathrm{THz}$ sources is lead by selfheating $[10,28,29]$. The S-shaped current-voltage curve can lead to two working points for the same resonance condition. In recent work it is proposed that, the high-bias regime may be more advantageous for $\mathrm{THz}$ emission than the low-bias regime, just because, in principal, emission can be reversibly scanned for increasing and decreasing bias in the high-bias regime. However, in the low-bias regime re-trapping events may prevent emission. Moreover, the emission linewidth is intrinsically lower at high biases than at low biases [30], and the emission frequency can be tuned by more than $30 \%$ for certain mesa geometries. At high bias, where heating seriously affects the local mesa temperature, back bending at the I-V curve is observed because of high critical current density and the large volume of the mesa. Temperature rise is directly related to the power generated in mesa structures $(\Delta \mathrm{T} \alpha \mathrm{P}=$ IV). The current is proportional to the junction area, and the voltage is proportional to the number of IJJs in the stack. Hence, in order to decrease the amount of heating, it is helpful to reduce either of these parameters to a proper doping level.

The coupling between a.c. Josephson effect and the cavity resonance conditions correlates with the tunability property of the radiation frequency. It was noted in the $\mathrm{I}-\mathrm{V}$ characteristics of IJJ mesa that the hysteresis loop of the I-V curve is vitally based on the temperature. The aforementioned dependence leads to the feasibility of large voltage applications to the mesa at lower temperatures as well. Therefore, at a higher cavity excitation mode, the emission is observed when the mesa structure has some resonance conditions opposing the higher bias voltage. At low temperature, introducing maximum bias voltage is relevant to the dimension of the mesa and, consequently, higher excitation modes are feasible by taking into account the mesa size.

In this study the intention is to optimize critical current density and search crystal inhomogeneity to significantly enhance the control over the superconducting properties for powerful $\mathrm{THz}$ emission.

\section{Experimental details}

In this work, the experiments were initiated on as-grown Bi2212 single crystals which were grown using the travelling solvent floating zone technique. In order to obtain appropriate doping level, we annealed the high temperature superconducting Bi2212 single crystals under vacuum condition with different heat treatment recipes. The heat treatment duration is varied to change the $T_{c}$ and critical current of crystals.

In the annealing set up, Fig. 1, we have used a vacuum pump and a furnace. At first we have reduced the pressure of the quartz tube in which we have located the crystals up to $10^{-4}$ mbar using the turbomolecular pump. Afterwards we have operated the furnace which has been previously programmed to the desired temperature and time in order to establish annealing at different temperatures. For further processing, a single crystal of $\mathrm{Bi} 2212$ is glued onto a sapphire substrate from its smooth a-b surface by silver epoxy. In order to get a fresh and smooth surface on Bi2212, the crystal was then cleaved with an adhesive tape and Au layer with thickness of $100 \mathrm{~nm}$ was thermally deposited on the cleaved crystal surface immediately to prevent the chemical reactions. To obtain natural IJJ stacks with various size and height, mesas on Bi2212 crystals have been fabricated using e-beam lithography and argon ion beam etching techniques [19-21]. Because of the difficulties in making a contact on small areas of the mesa, first a $\mathrm{CaF}_{2}$ insulating layer is deposited by evaporation onto the crystal and a gold stripe is formed by e-beam lithography on the insulating layer and mesa. Finally three gold probe wires are connected on two pads and mesa by silver epoxy. After the mesa fabrication, the exact dimensions of the mesas were obtained using a surface profilometer and atomic force microscope. The number of Josephson junctions were determined which gives the emission voltage. The electrical characterization of the mesas was obtained from room temperature through low temperatures. In order to characterize the Bi2212 mesas, c-axis resistance versus temperature $(\mathrm{R}-\mathrm{T})$, and current-voltage behavior $(\mathrm{I}-\mathrm{V})$ were measured in a He flow cryostat.

\section{Results and discussion}

The scanning electron microscope (SEM) images of the sample are shown in Fig. 2 as an example. It is noted that the sample is not rectangular but has a considerable trapezoidal shape after ion beam etching. The dark shaded area on the top of the mesa structures is $\mathrm{CaF}_{2}$ layer is for electrical insulation purpose. We see the gold wires that are attached to the gold strip structures in Fig. 2(b), (d). From the previous studies, it is demonstrated that the side of the mesa is a very important parameter since it leads to impedance mismatch between Bi2212 and vacuum.

Resistance versus temperature $(\mathrm{R}-\mathrm{T})$ characteristics along the caxis of the single Bi2212 $50 \times 300 \mu \mathrm{m}^{2}$ mesa (Sample A) was 


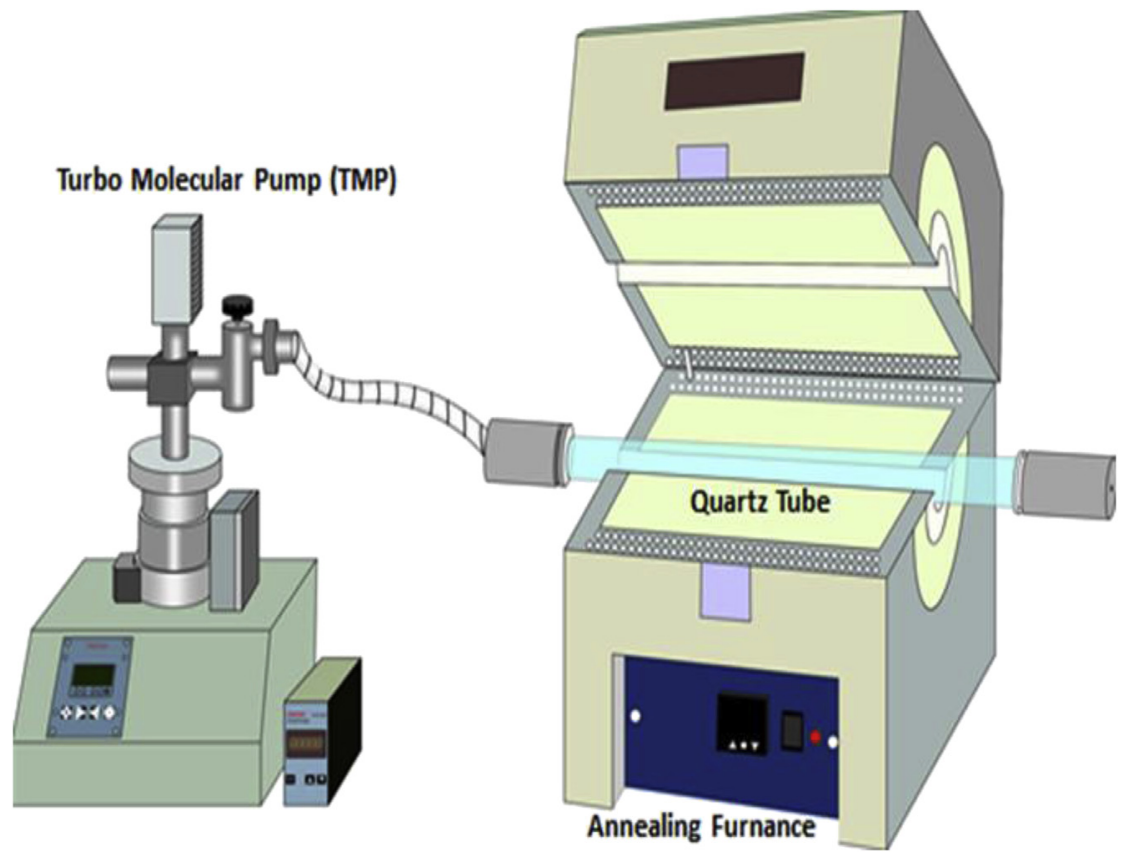

Fig. 1. Annealing system in vacuum.

measured in a He flow cryostat system. During the measurement a constant $10 \mu \mathrm{A}$ current is applied along the mesa. As seen in Fig. 3(a) both temperature dependence of the c-axis resistance and the superconducting transition temperature $\left(T_{c}=86.5 \mathrm{~K}\right)$ of the mesa structure indicate that it is in the under-doped state of the Bi2212 crystals which is suitable for terahertz emission. The ratio of $R\left(T_{c}\right) / R(300 \mathrm{~K})$ is 4.1 for this mesa structure. Watanabe et al. have systematically studied the oxygen doping level dependence of the c-axis resistivity of Bi2212 and the range $\mathrm{R}\left(\mathrm{T}_{\mathrm{C}}\right) / \mathrm{R}(300 \mathrm{~K})>4$ indicates a necessity of a certain doping range of $\partial \sim 0.22$ for $\mathrm{THz}$ emission [31,32]. This shows the importance of oxygen doping level for $\mathrm{THz}$ emission.

The temperature dependence of c-axis tunneling characteristic of Sample A is seen in Fig. 3(b). The tunneling behavior goes from the superconducting state to normal state from temperatures $15-30 \mathrm{~K}$. In the superconducting I-V data, it is generally seen that

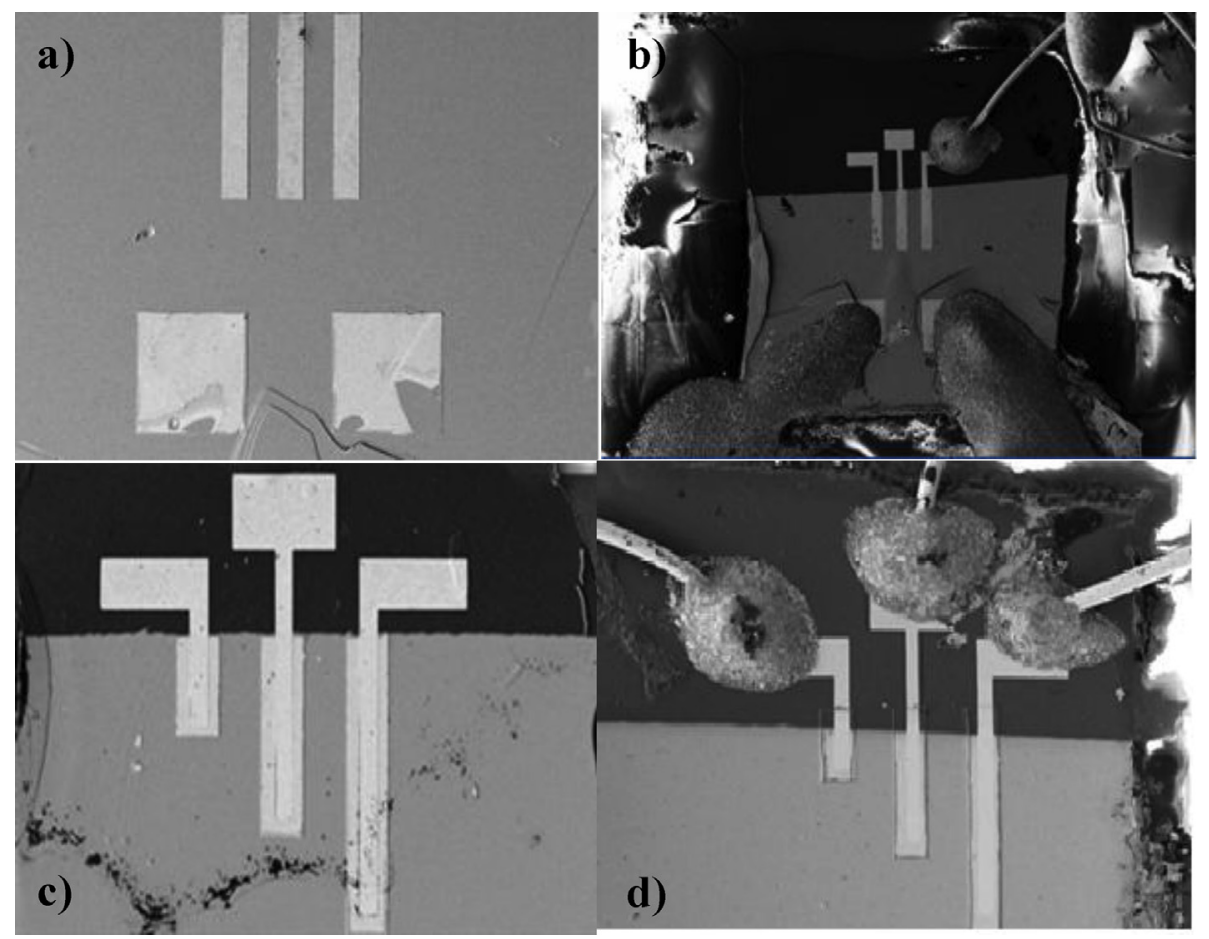

Fig. 2. (a), (b) SEM images of $300 \times 50 \mu \mathrm{m}^{2}$ triple mesa structures fabricated on Bi2212 crystal with different magnifications. (c), (d) SEM images of $300 \times 50 \mu \mathrm{m}^{2}$ triple mesa structures fabricated on Bi2212 crystal with different magnifications. 

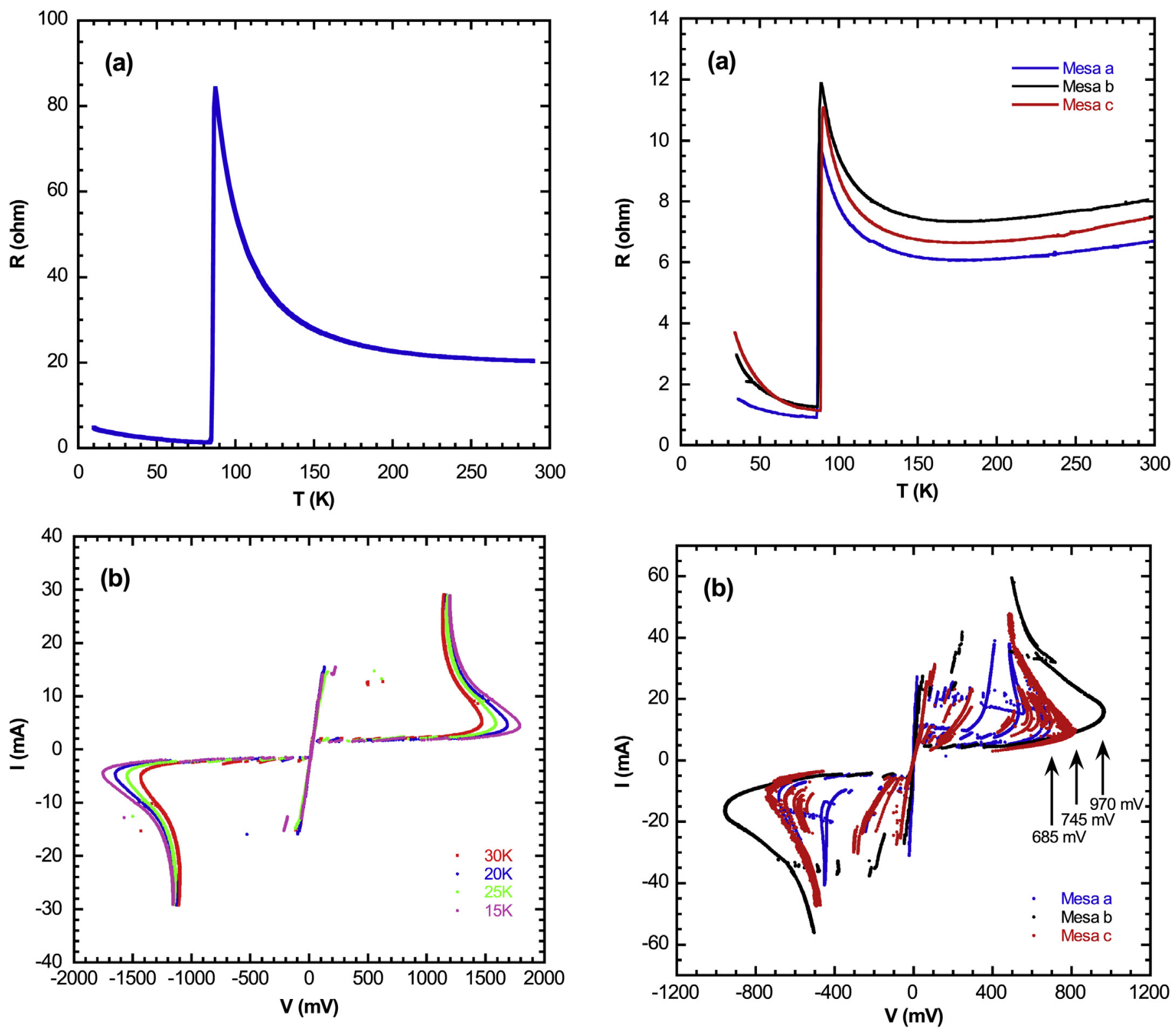

Fig. 3. (a) R-T measurements of $300 \times 50 \mu \mathrm{m}^{2}$ single mesa structure. (b) Temperature evolution of $\mathrm{I}-\mathrm{V}$ measurement of $300 \times 50 \mu \mathrm{m}^{2}$ single mesa structure.

total conductance peak value decreases with increasing temperature. This is compatible with the temperature dependence of the energy gap in the BCS theory. For this reason, decreasing the individual energy gap reveals a reduction in total conductance peak of IJJ stacks. This could be intrinsically observed in spite of the presence of Joule heating in a large number of IJJs. From the bolometric detections, there is no sign of $\mathrm{THz}$ emission although we observed the increasing detection level of radiation at higher currents and voltages, which would be originated by the thermal radiation due to the sample heating.

The curves in Fig. 4(a) exhibit a typical temperature dependence of the c-axis resistance of the triple mesa structure of Bi2212 (Sample B), which tends to increase with decreasing temperature around room temperature and turns to a sharp increase near $T_{c}$. Triple mesa structures of the Sample $B$ have the same area of $50 \times 300 \mu \mathrm{m}^{2}$. Mesa resistance is finite even below $\mathrm{T}_{\mathrm{c}}$. This is because of the contact resistance due to the three terminal

Fig. 4. (a) R-T measurements of $300 \times 50 \mu \mathrm{m}^{2}$ triple mesa structure on the same crystal $100 \mu \mathrm{m}$ apart from each other. (b) I-V Characteristics of $300 \times 50 \mu \mathrm{m}^{2}$, $200 \times 50 \mu \mathrm{m}^{2}, 100 \times 50 \mu \mathrm{m}^{2}$ triple mesa structure on same crystal at $20 \mathrm{~K}$.

measurements. However, the contact resistance is usually smaller than a few ohms so that it seems not to influence the measurement, but it may contribute to the heating, which will be discussed below. Our samples so far prepared have $\mathrm{T}_{\mathrm{c}}$ between 75 and $90 \mathrm{~K}$, which indicates that the mesa is in the nearly under-doped condition. I-V characteristic of Bi2212 mesas show a number of characteristic features of the multi stacked intrinsic Josephson junction. They include several branches due to individual switching of each junction from Josephson state to resistive state when the bias current exceeds the individual critical current of each junction.

Fig. 4(b) shows a comparison of I-V measurements of Bi2212 mesa structures of Sample B. Although they are on the same crystal, the backbending voltage and critical current values of the mesa structures whose critical temperatures exhibit discrepancy are different from each other due to the inhomogeneity of the crystal. We can also observe from the transitions in resistance temperature 
graphics that the crystal is inhomogeneous. In non-uniform mesas the doping levels of the intrinsic Josephson junctions could be slightly different. In the triple mesa structures of Sample $B$ for which we have changed doping, critical current densities $\left(J_{c}=I_{c} / A\right)$ are $180,186,200 \mathrm{~A} / \mathrm{cm}^{2}$ for $50 \times 300 \mu \mathrm{m}^{2}$, respectively. The critical current of the branches increases monotonically, which shows that the doping along the crystal is inhomogeneous. Back-bending of the $\mathrm{I}-\mathrm{V}$ curves (that is, a negative differential resistance) at high current biases is a clear indication of self-heating. The rise in mesa temperature and the rapid decrease in the c-axis resistance with increasing temperature result in the back-bending of the $\mathrm{I}-\mathrm{V}$ curves.

Fig. 5(a) and (b) represent the R-T and I-V measurement of Sample $\mathrm{C} 1$, which is annealed at $450{ }^{\circ} \mathrm{C}$ for $4 \mathrm{~h}$. By the annealing procedure that we performed in our experiments, we can change the superconducting properties as well as the critical supercurrent
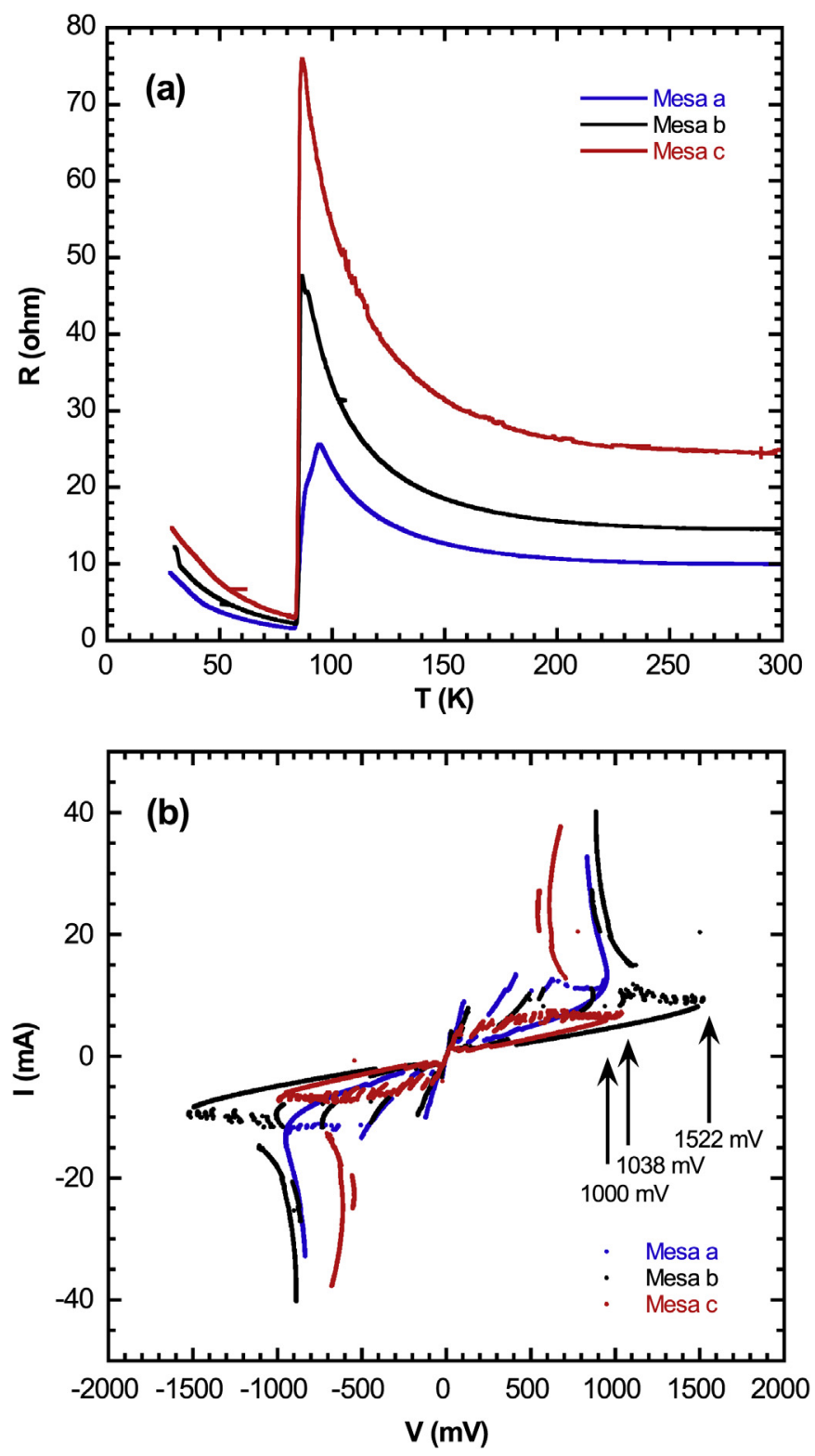

Fig. 5. (a) R-T measurements of $300 \times 50 \mu \mathrm{m}^{2}, 200 \times 50 \mu \mathrm{m}^{2}, 100 \times 50 \mu \mathrm{m}^{2}$ triple mesa structures on the same crystal $100 \mu \mathrm{m}$ apart from each other. (b) I-V Characteristics of $300 \times 50 \mu \mathrm{m}^{2}, 200 \times 50 \mu \mathrm{m}^{2}, 100 \times 50 \mu \mathrm{m}^{2}$ triple mesa structures on same crystal at $20 \mathrm{~K}$.
Table 1

Critical current, backbending point and calculated critical current density values of the triple mesa structures.

\begin{tabular}{llrcl}
\hline Sample ID & $\begin{array}{l}\text { Mesa dimensions } \\
\left(\mu \mathrm{m}^{2}\right)\end{array}$ & $\mathrm{I}_{\mathrm{c}}(\mathrm{mA})$ & $\mathrm{J}_{\mathrm{c}}\left(\mathrm{A} / \mathrm{cm}^{2}\right)$ & $\begin{array}{l}\text { Backbending } \\
\text { point }(\mathrm{mV})\end{array}$ \\
\hline SampleC1a & $300 \times 50$ & 10.1 & 60 & 1000 \\
SampleC1b & $200 \times 50$ & 8.1 & 80 & 1522 \\
SampleC1c & $100 \times 50$ & 5.0 & 100 & 1038 \\
SampleC2a & $300 \times 50$ & 21.8 & 140 & 720 \\
SampleC2b & $200 \times 50$ & 18.4 & 160 & 760 \\
SampleC2c & $100 \times 50$ & 9.0 & 180 & 1120 \\
SampleC3a & $300 \times 50$ & 12.0 & 80 & 1555 \\
SampleC3b & $200 \times 50$ & 15.5 & 155 & 1750 \\
SampleC3c & $100 \times 50$ & 10.0 & 400 & 2000 \\
\hline
\end{tabular}

density of an intrinsic junction stack. The area of mesa a, mesa b and mesa $c$ structures of Sample C1 are $300 \times 50 \mu^{2}$, $200 \times 50 \mu \mathrm{m}^{2}, 100 \times 50 \mu \mathrm{m}^{2}$ respectively.

It is already shown that the $\mathrm{THz}$ emitting mesas are below a certain under-doped level, which has relatively small critical current in contrast to optimally doped and over-doped Bi2212 [31,32]. Because of the small critical current, large area mesas fabricated from under-doped crystals cause less heating and backbending occurs after the cavity resonance in the voltage scale. So, Powerful $\mathrm{THz}$ radiation can be obtained before heating severely affects the local mesa temperature. Especially, mesa with area of $100 \times 50 \mu \mathrm{m}^{2}$, shows many quasiparticle branches in Fig. 5(b). We can see that the critical current of the fabricated mesas are 10.03, 8.05 and $5.03 \mathrm{~mA}$ for mesa a, mesa b and mesa c of Sample C1, respectively (Table 1$)$. Therefore, we can calculate the Josephson critical current densities as 60,80 and $100 \mathrm{~A} / \mathrm{cm}^{2}$ for mesa a, mesa b and mesa $\mathrm{c}$ of Sample C1. However, because it has fairly poor thermal conductivity, intrinsic junctions of Bi2212 suffer from selfheating at high bias voltages. In addition, heating is relatively larger for tunneling into an IJJ stack than a conventional superconducting junction on Bi2212 because of lower specific junction resistance caused by its very thin barrier. As a result of self-heating in IJJ mesas, some artifacts have been observed in the tunneling characteristics.

By increasing the number of IJJs in the quasiparticle state, the overheating increases and leads to a smaller voltage jump (conductance peak) in $\mathrm{I}-\mathrm{V}$ curves. Furthermore, the nonequilibrium effect, which is easy quasiparticle self-injection through the high transmissive IJJ tunneling barrier (about $0.3 \mathrm{~nm}$ thickness in Bi2212) increases the heating effect in the structure. This effect also leads to back-bending of the I-V curve in the subgap region. One of the methods to reduce the problem is to use stacks of intrinsic junctions (mesas) with small dimensions in order to reduce $\mathrm{I}_{\mathrm{c}}$. However, since the same current per unit area through the junctions flows, the non-equilibrium effect still occurs and results in the Joule heating.

Several groups have attempted different approaches to the overheating problem such as reduction of the current density by intercalation of some molecules such as $\mathrm{HgB}_{2}, \mathrm{HgI}_{2}, \mathrm{I}_{2}$ within the $\mathrm{Bi}-\mathrm{O}$ bilayer, which increase the c-axis resistance [33,34]. In addition, using short pulses and varying the mesa dimensions are other methods to decrease the self-heating effects. In all I-V curves, the large current density and close proximity of neighboring junctions in Bi2212 junction arrays are interpreted as the cause of such heating effects. In 2006 Zhu et al. proposed a study about the junction-size dependence of self-heating for ultra-small mesa structures [35]. It is demonstrated that self-heating decreases systematically with the reduction of junction area. In this study, the dependence of Josephson critical current density on the mesa area 
for large area mesa structures on the same chip is shown. As a consequence, self-heating depends on the mesa size and the number of junctions.

\section{Conclusion}

In this study, we presented the R-T and I-V characteristics of triple mesa structures fabricated on Bi2212 crystals. We observed hysteresis type quasiparticle branches in the performed I-V measurements. Heating effects are observed on the current-voltage curves due to the large volume of the mesas. A self heating effect leads to de-synchronization and consequently there will be a dramatic drop in $\mathrm{THz}$ emission power. It is found that the critical current density is decreasing with increasing mesa area and hence backbending is reduced. Since the generation of powerful terahertz emission needs low critical current density and reduces the heating effect, the estimated heat reduction can be improved with optimized mesa area. These results can be applied to the mesas to fill the so-called terahertz gap using superconducting terahertz sources. It is also shown that the Josephson current density and backbending point obtained from mesa structures on the same crystal can be strongly influenced by inhomogeneous doping, defects and impurities. It is necessary to have perfect crystal structure with homogeneous doping to generate powerful $\mathrm{THz}$ emission.

\section{Acknowledgements}

This work has been supported in part by TUBITAK (Scientific and Technological Council of Turkey) under project number 110T248. We would also like to acknowledge SANTEZ project number 1386.STZ.2012-1. The infrastructure of Applied Quantum Research Center (UKAM) was used to perform some parts of the experiments.

\section{References}

[1] Tonouchi M. Cutting-edge terahertz technology. Nat Photonics 2007;97:105.

[2] Ferguson B, Zhang XC. Materials for terahertz science and technology. Nat Mater 2006;1:26-33.

[3] Chan WL, Deibel J, Mittleman DM. Imaging with terahertz radiation. Rep Prog Phys 2007;70:1325-79.

[4] Kleine-Ostmann T, Nagatsuma T. A review on terahertz communications research. J Infrared Millim Te Waves 2011;32:143-71.

[5] Siegel PH. Terahertz technology in biology and medicine. IEEE Trans Microw Theory Tech 2004;52:2438-47.

[6] Ozyuzer L, Koshelev AE, Kurter C, Gopalsami N, Li Q, Tachiki M, et al. Emission of coherent THz radiation from superconductors. Science 2007;318:1291.

[7] Bae MH, Lee HJ, Choi JH. Josephson-vortex-flow terahertz emission in layered high-Tc superconducting single crystals. Phys Rev Lett 2007;98:027002.

[8] Koshelev AE, Bulaevskii LN. Resonant electromagnetic emission from intrinsic Josephson-junction stacks with laterally modulated josephson critical current. Phys Rev B 2008;77:014530.

[9] Hu X, Lin S. Three-dimensional phase-kink state in a thick stack of Josephson junctions and terahertz radiation. Phys Rev B 2008;78:134510-1.

[10] Benseman TM, Koshelev AE, Gray KE, Kwok W-K, Welp U, Kadowaki K, et al. Tunable terahertz emission from $\mathrm{Bi}_{2} \mathrm{Sr}_{2} \mathrm{CaCu}_{2} \mathrm{O}_{8+\delta}$ mesa devices. Phys Rev B 2011;84:064523.

[11] Hu X, Lin SZ. Phase dynamics in a stack of inductively coupled intrinsic Josephson junctions and terahertz electromagnetic radiation. Supercond Sci Technol 2010;23:053001.
[12] Krasnov VM. Terahertz electromagnetic radiation from intrinsic Josephson junctions at zero magnetic field via breather-type self-oscillations. Phys Rev B 2011;83:174517.

[13] Kashiwagi T, Tsujimoto M, Yamamoto T, Minami H, Yamaki K, Delfanazari K, et al. High temperature superconductor terahertz emitters: fundamental physics and its applications. Jpn J Appl Phys 2012;51:010113.

[14] Kleiner R, Steinmeyer F, Kunkel G, Müller P. Intrinsic Josephson effects in $\mathrm{Bi}_{2} \mathrm{Sr}_{2} \mathrm{CaCu}_{2} \mathrm{O}_{8}$ single crystals. Phys Rev Lett 1992;68:2394.

[15] Tachiki M, Iizuka M, Minami K, Tejima S, Nakamura H. Emission of continuous coherent terahertz waves with tunable frequency by intrinsic Josephson junctions. Phys Rev B 2005;71:134515-1-134515-5.

[16] Klemm RA, LaBerge ER, Morley DR, Kashiwagi T, Tsujimoto M, Kadowaki K. Cavity mode waves during terahertz radiation from rectangular $\mathrm{Bi}_{2} \mathrm{Sr}_{2} \mathrm{CaCu}_{2} \mathrm{O}_{8+\delta}$ mesas. J Phys Cond Matt. 2011;23:025701.

[17] Tsujimoto M, Yamaki K, Deguchi K, Yamamoto T, Kashiwagi T, Minami H, et al. Geometrical resonance conditions for $\mathrm{THz}$ radiation from the intrinsic Josephson junctions in $\mathrm{Bi}_{2} \mathrm{Sr}_{2} \mathrm{CaCu}_{2} \mathrm{O}_{8+\delta}$. Phys Rev Lett 2010;105: 037005.

[18] Kadowaki K, Tsujimoto M, Yamaki K, Yamamoto T, Kashiwagi T, Minami H et al. Evidence for a dual-source mechanism of terahertz radiation from rectangular mesas of single crystalline $\mathrm{Bi}_{2} \mathrm{Sr}_{2} \mathrm{CaCu}_{2} \mathrm{O}_{8+\delta}$ intrinsic Josephson junctions. J Phys Soc Jpn 2010;79:023703.

[19] Ozyuzer L, Simsek Y, Koseoglu H, Turkoglu F, Kurter C, Welp U, et al. Terahertz wave emission from intrinsic Josephson junctions in high-Tc superconductors. Supercond Sci Technol 2009;22:114009.

[20] Turkoglu F, Koseoglu H, Demirhan Y, Ozyuzer L, Preu S, Malzer S, et al. Interferometer measurements of terahertz waves from $\mathrm{Bi}_{2} \mathrm{Sr}_{2} \mathrm{CaCu}_{2} \mathrm{O}_{8+\mathrm{d}}$ mesas. Supercond Sci Technol 2012;25:125004.

[21] Turkoglu F, Ozyuzer L, Koseoglu H, Demirhan Y, Preu S, Malzer S, et al. Emission of the $\mathrm{THz}$ waves from large area mesas of superconducting $\mathrm{Bi}_{2} \mathrm{Sr}_{2} \mathrm{CaCu}_{2} \mathrm{O}_{8+\mathrm{d}}$ by the injection of spin polarized current. Phys C Supercond 2013;491:7.

[22] Wang HB, Guénon S, Yuan J, Iishi A, Arisawa S, Hatano T, et al. Hot spots and waves in $\mathrm{Bi}_{2} \mathrm{Sr}_{2} \mathrm{CaCu}_{2} \mathrm{O}_{8}$ intrinsic josephson Junction stacks- a study by low temperature scanning laser microscopy. Phys Rev Lett 2009;102: 017006.

[23] Wang HB, Guénon S, Gross B, Yuan J, Jiang ZG, Zhong YY, et al. Coherent terahertz emission of intrinsic josephson Junction stacks in the hot spot regime. Phys Rev Lett 2010;105:057002.

[24] Guénon S, Grünzweig M, Gross B, Yuan J, Jiang Z, Zhong Y, et al. Interaction of hot spots and $\mathrm{THz}$ waves in $\mathrm{Bi}_{2} \mathrm{Sr}_{2} \mathrm{CaCu}_{2} \mathrm{O}_{8}$ intrinsic Josephson junction stacks of various geometry. Phys Rev B 2010;82:214506.

[25] Kurter C, Ozyuzer L, Proslier T, Zasadzinski JF, Hinks DG, Gray KE. Counterintuitive consequence of heating in strongly-driven intrinsic junctions of $\mathrm{Bi}_{2} \mathrm{Sr}_{2} \mathrm{CaCu}_{2} \mathrm{O}_{8+\delta}$ mesas. Phys Rev B 2010;81:224518.

[26] Kurter C, Gray KE, Zasadzinski JF, Ozyuzer L, Koshelev AE, Li Q et al. Thermal management in large Bi2212 mesas used for terahertz sources. IEEE Trans Appl Supercond 2009;19:428.

[27] Krasnov VM, Sandberg M, Zogaj I. In situ measurement of self-heating in intrinsic tunneling spectroscopy. Phys Rev Lett 2005;94:077003.

[28] Krasnov VM. Comment on "Counterintuitive consequence of heating in strongly-driven intrinsic junctions of $\mathrm{Bi}_{2} \mathrm{Sr}_{2} \mathrm{CaCu}_{2} \mathrm{O}_{8}$ mesas". Phys Rev B 2011;84:136501.

[29] Welp U, Kadowaki K, Kleiner K. Superconducting emitters of THz radiation. Nat Photonics 2013;7:702.

[30] Li M, Yuan J, Kinev N, Li J, Gross B, Guénon S, et al. Linewidth dependence of coherent terahertz emission from $\mathrm{Bi}_{2} \mathrm{Sr}_{2} \mathrm{CaCu}_{2} \mathrm{O}_{8}$ intrinsic Josephson junction stacks in the hot-spot regime. Phys Rev B 2012;86:060505.

[31] Koval Y, Jin X, Bergmann C, Simsek Y, Ozyuzer L, Müller P, et al. Tuning superconductivity by carrier injection. Appl Phys Lett 2010;96:082507.

[32] Watanabe T, Fuji T, Matsuda A. Anisotropic resistivities of precisely oxygen controlled single-crystal $\mathrm{Bi}_{2} \mathrm{Sr}_{2} \mathrm{CaCu}_{2} \mathrm{O}_{8+\delta}$ : systematic study on "Spin Gap" effect. Phys Rev Lett 1997;79:2113.

[33] Ozyuzer L, Kurter C, Ozdemir M, Zasadzinski JF, Gray KE, Hinks DG. Spin polarized current injection through $\mathrm{HgBr}_{2}$ Intercalated $\mathrm{Bi} 2212$ intrinsic josephson junctions. IEEE Trans Appl Supercond 2007; 17:577.

[34] Ozyuzer L, Ozdemir M, Kurter C, Hinks DG, Gray KE. Effect of magnetic field on quasiparticle branches of intrinsic Josephson junctions with ferromagnetic layer. Phys C Supercond 2007;950:460-2.

[35] Zhu XB, Wei YF, Zhao SP, Chen GH, Yang HF, Jin AZ, et al. Intrinsic tunneling spectroscopy of $\mathrm{Bi}_{2} \mathrm{Sr}_{2} \mathrm{CaCu}_{2} \mathrm{O}_{8+\delta}$ the junction-size dependence of self-heating. Phys Rev B 2006;73:224501. 